\title{
Forschung in Palliative Care: Die Schweiz holt auf
}

\author{
Myriam Tapernouxa , Sarah Fasolin ${ }^{b}$
}

${ }^{a}$ Leiterin Ressort Wissenschaft SAMW; ${ }^{b}$ Freie Journalistin

Viele Menschen brauchen auf ihrem letzten Stück Weg palliative Behandlung. Doch ausgerechnet in diesem für Medizin und Gesellschaft wichtigen Thema hinkte die Schweiz anderen Ländern hinterher, auch in der Forschung. Unter dem Titel «Forschung in Palliative Care 2014-2018» lancierte die Schweizerische Akademie der Medizinischen Wissenschaften (SAMW) deshalb ein Förderprogramm, das insgesamt 4,4 Millionen Franken ausschütten konnte. Der Schlussbericht zeigt, was es gebracht hat.

Gerade einmal 12 Publikationen zum Thema Palliative Care in der Schweiz zählte die Genfer Professorin Sophie Pautex im Jahr 2008. Pautex ist Mitgründerin der «Palliative Care Forschungsplattform Schweiz» und war Mitglied der Evaluationskommission des SAMW-Förderprogramms. 12 Publikationen sind nicht überragend angesichts der Relevanz, die Palliative Care im medizinischen Alltag einnimmt. Denn: In der Schweiz sterben heute rund 90\% der Bevölkerung nicht aufgrund eines plötzlichen Todes, sondern nach einer Krankheits- und Pflegephase. Das Ende des Lebens ist somit bei den meisten Menschen ein unterschiedlich lang dauernder Prozess.

\footnotetext{
Das Wichtigste in Kürze

Palliative Care in der Schweiz ist im medizinischen Alltag allgegenwärtig und wird aufgrund der demographischen Entwicklung immer relevanter.

Mit dem SAMW-Programm «Forschung in Palliative Care 2014-2018», das von der Stanley Thomas Johnson Stiftung und der Gottfried und Julia Bangerter-Rhyner Stiftung finanziert wurde, konnten 34 Projekte gefördert und vier Stipendien vergeben werden.

Das Förderprogramm warTeil einer von Bund und Kantonen angestossenen Forschungsoffensive, die in der Schweiz den Rückstand in Palliative Care zu anderen Ländern aufholen sollte.

Ein von der SAMW organisiertes Abschlusssymposium, an dem 120 palliativ arbeitende Professionelle teilnahmen zeigte: Es konnte viel erreicht werden, braucht aber weiter hin Kontinuität und Finanzen.
}

Nicht nur die Forschungstätigkeit war in der Schweiz vor gut zehn Jahren unzureichend, auch das Angebot für Patientinnen und Patienten. Bund und Kantone lancierten deshalb die «Nationale Strategie Palliative

Insgesamt wurden 138 Anträge geprüft, 34 Projekte und vier Stipendien mit total 4,4 Mio. Franken finanziert.

Care 2010-2015». Ziel war, das gesamte Spektrum der Palliative Care zu fördern: Sensibilisierung, Versorgung, Bildung, Finanzierung und Forschung. Damit verbunden stiess der Bundesrat das «Nationale Forschungsprogramm Lebensende» (NFP 67) an, und die SAMW führte das Förderprogramm «Forschung in Palliative Care 2014-2018» durch.

Insgesamt wurden 138 Anträge geprüft, 34 Projekte finanziert und vier Stipendien für Ausbildungs- und Forschungsaufenthalte im Ausland gesprochen. Die Onkologie wurde bewusst ausgeklammert, da Forschung in diesem Bereich bereits anderweitig finanziert wird. Die Projekte beschäftigten sich mit Fragen zur Behandlung von körperlichen Symptomen (Wie können Schmerzen gelindert werden?), mit Themen zur psychosozialen und spirituellen Begleitung (Wie findet man z.B. Antworten auf den Sinn des Lebens? Wie werden Angehörige einbezogen?), mit ethischen Fragen (Welche Entscheidungen, z.B. Freitod, sind möglich und vom Betroffenen gewünscht?), aber auch mit infrastrukturellen und prozeduralen Aspekten (Soll der Patient zu Hause oder stationär betreut werden?). 
Um Antworten auf solche Fragen zu finden, konnte die SAMW zwei private Stiftungen gewinnen: die Stanley Thomas Johnson Stiftung und die Gottfried und Julia Bangerter-Rhyner Stiftung unterstützten das Förderprogramm mit insgesamt 4,4 Millionen Franken.

\section{Bessere Datenlage, stärkere Netzwerke}

Eine abschliessende Bilanz kann zum heutigen Zeitpunkt nicht gezogen werden, da einige Projekte noch laufen. Doch bereits jetzt lässt sich festhalten, dass das Förderprogramm die Datenlage in der Palliative Care wesentlich verbessern konnte. Zudem sind Forschungsnetzwerke entstanden, der Austausch unter Spitälern und anderen Institutionen ist intensiver geworden, und es sind zahlreiche Publikationen hervorgegangen. Nennenswert ist auch

\section{Die demographische Entwicklung zeigt: Die Bevölkerung wird immer älter. Somit wird auch Palliative Care immer wichtiger.}

die Verbesserung im Nachwuchsbereich: Junge Forschende profitierten vom Programm entweder als Mitarbeitende von Projekten oder indem sie direkt mit einem Fellowship gefördert wurden.
Mit dem Symposium "Research in Palliative Care» wurde am 21. November 2019 in Bern das Förderprogramm offiziell abgeschlossen. Rund 120 Professionelle, die in ihrem Alltag mit Palliative Care zu tun haben, beschäftigten sich einen Tag lang mit diversen Fragen rund um Forschung am Lebensende. Resultate wurden präsentiert, aber auch Herausforderungen thematisiert, denen man als Forscher gegenübersteht. Immer wieder kam am Symposium zum Ausdruck: Die Forschung zu Palliative Care in der Schweiz muss wei-

Forschung in Palliative Care muss institutionell verankert werden, um für Forschungsnachwuchs attraktiv zu sein.

tergehen, denn ein Blick in die Zukunft zeigt: Palliative Care wird uns je länger, je mehr beschäftigen, weil die Bevölkerung immer älter wird. Gemäss dem Bundesamt für Statistik beträgt der Anteil der Bevölkerung $60+$ in der Schweiz heute $25,4 \%$. Im Jahr 2050 werden es bereits $33,8 \%$ sein.

ie Publikation «Forschung in Palliative Care - Schlussberich zum SAMW-Förderprogramm 2014-2018" ist kostenlos gedruck oder als Download erhältlich: samw.ch/de/palliative-care

\section{Einblick in ausgewählte Projekte}

In der Palliative Care arbeiten verschiedene Berufsgruppen eng zusammen. Gleichzeitig beziehen sie das persönliche Umfeld mit ein, damit der oder die Betroffene bis zum Tod eine bestmögliche Lebensqualität erhält. Diese thematische Breite zeigte sich auch in der Vielfalt der Forschungsfragen, die im Rahmen des SAMWFörderprogramms untersucht wurden.

\section{Auf der Suche nach einer Datenbasis}

Wer sind eigentlich die Palliative-Care-Patienten in der Schweiz? Wie läuft das Leben einer Patientin innerhalb von entsprechenden Institutionen genau ab? Um solchen Fragen auf den Grund zu gehen, schickte ein Forschungsteam um Steffen Eychmüller vom Inselspital Bern und Sophie Pautex vom Unispital Genf Fragebögen an sechs PalliativeCare-Abteilungen in der Schweiz. 379 Bögen konnten ausgewertet werden: Die in einer Palliative-Care-Abteilung behandelte Person war im Durchschnitt 73 Jahre alt, weiblich, protestantisch und an Krebs erkrankt. Nicht-KrebsPatienten haben es schwieriger, an Palliative-Care-Angebote zu kommen, wie aufgrund der Studie deutlich wurde. Wichtig war den Forschenden auch, zeigen zu können, dass die Etablierung einer nationalen Datenbasis für Palliative Care möglich wäre. Das Projekt soll deshalb weiter vorangetrieben werden mit dem Ziel, ein entsprechendes Datentool in der Schweiz zu institutionalisieren.

\section{Wenn Menschen aufhören, zu essen und zu trinken}

Die meisten waren um die 80 Jahre alt und litten an Krebs oder anderen lebensbedrohlichen Krankheiten. Ihre Lebenserwartung betrug weniger als ein Jahr. Weil sie Angst davor hatten, von anderen abhängig zu werden, und müde 
und erschöpft waren, entschieden sie, mit Essen und Trinken aufzuhören. In den meisten Fällen verstarben die Betroffenen nach rund 13 Tagen. Dies sind einige der Resultate einer ersten nationalen Befragung zum Freiwilligen Verzicht auf Nahrung und Flüssigkeit (FVNF), die ein Forscherteam um André Fringer von der Zürcher Hochschule für Angewandte Wissenschaften in einer empirischen Studie erhoben hat. Zwar ist FVNF im beruflichen Alltag eine Randerscheinung - nur gerade 1,7\% der Patienten, die in Schweizer Pflegeeinrichtungen sterben, wählen diesen Weg. Für involvierte Professionelle kann der FVNF jedoch eine grosse Herausforderung sein und sie in ein ethisches Dilemma bringen. Anhand der Studienergebnisse und der bereits existierenden wissenschaftlichen Erkenntnisse zum FVNF ist gemäss den Forschenden deutlich geworden, «dass eine professionelle Auseinandersetzung z.B. mittels Schulungsmassnahmen zum FVNF notwendig ist».

\section{Palliative Care zu Hause bei ALS-Patienten}

Amyotrophe Lateralsklerose (ALS) ist eine degenerative Krankheit des Nervensystems, für die es keine Heilung gibt. Von der Diagnose bis zum Tod bleiben ALS-Patienten meistens zwei bis drei Jahre Lebenszeit. Oft werden ALSPatienten zu Hause von einzelnen oder mehreren Angehörigen bis zum Tod gepflegt. Ein Forschungsteam um Tenzin Wangmo vom Institut für Bio- und Medizinethik an der Universität Basel will herausfinden, was für Bedürfnisse pflegende Angehörige haben und wie sie in dieser emotional, aber auch körperlich herausfordernden Zeit unterstützt werden können.

\section{Dankbar werden für ein zu Ende gehendes Leben}

Wer seinen Angehörigen gegenüber dankbar sein kann, geht das letzte Stück Weg zufriedener und gestärkt. Davon gehen die Forschenden um Mathieu Bernard vom Universitätsspital Lausanne aus. Denn aus früheren Forschungsprojekten ist bekannt, dass nicht-physische Faktoren einen grossen Einfluss auf die Lebensqualität eines Patienten und seiner Familie haben können. Wie aber wird man dankbar? Wie kann man Menschen dabei unterstützen? Für ihre Studie führen die Forscher mit 30 Patienten-Angehörigen-Paaren Dankbarkeits-Interventionen durch. Eine Intervention besteht aus zwei Schritten. Der erste ist ein Dankbarkeitsbrief, in dem Patient und Angehörige jeweils schreiben, weshalb sie dem anderen gegenüber dankbar sind. Der zweite Schritt ist ein Dankbarkeitsbesuch, während dessen einander die Briefe überreicht und vorgelesen oder zum späteren Lesen mitgegeben werden.

\section{Herzoperation und/oder Palliative Care?}

Ältere Patienten mit symptomatischer Aortenstenose müssen mit einer stark erhöhten Sterblichkeit rechnen. Eine Forschungsgruppe um Tanja Krones vom UniversitätsSpital Zürich versucht mittels Interviews herauszufinden, welche Wünsche diese Patienten für die aktuelle Behandlungsplanung oder bei zukünftigen gesundheitlichen Krisen haben. Möchte sich der Patient einer offenen Herzoperation oder einer weniger invasiven kardialen Intervention unterziehen lassen? Zu welchem Zeitpunkt möchte er den Fokus auf einen palliativen Behandlungsplan legen? Wie soll insbesondere im Falle von Komplikationen während oder nach der Operation vorgegangen werden? Eine Analyse der Interview-Aussagen soll als Entscheidungshilfe aufgearbeitet werden. Mit diesem Instrument soll es dem Patienten, den Angehörigen und den interprofessionellen Behandlungsteams leichter fallen, einen stimmigen Weg zwischen den medizinischen Möglichkeiten und den zu erwartenden Chancen und Komplikationen zu finden.

\section{Tod am Lebensanfang}

Jedes Jahr sterben in der Schweiz rund 600 Babys im Zeitraum zwischen der 22. Schwangerschaftswoche und der ersten Woche nach der Geburt. Diese perinatalen Todesfälle sind für die betroffenen Eltern ein äusserst schmerzhaftes Erlebnis, das im schlimmsten Fall zu posttraumatischen Belastungsstörungen, Depressionen und anderen Problemen führen kann. Eine Forschungsgruppe um Claudia Meier Magistretti von der Hochschule Luzern und Valerie Fleming von der Liverpool University eruierte mittels Interviews mit betroffenen Eltern, welche Betreuung und Unterstützung hilft, damit möglichst keine psychischen Langzeitfolgen entstehen. Ein von den Forschern gemeinsam mit Eltern, Fachpersonen und Krankenversicherungen entwickeltes Best-Practice-Modell hält diverse Massnahmen fest, die betroffenen Mütter und Väter vom Zeitpunkt der Diagnose bis zur Trauerverarbeitung wirksam unterstützen. 